\title{
Design and regulation of clinical trials; from the laboratory to the pharmacy
}

\author{
Article by Ekenechukwu E Young ${ }^{1}$ and Chinyere N Okafor ${ }^{2}$ \\ ${ }^{1}$ Department of Medicine, University of Nigeria, Enugu Campus. \\ ekenechukwu.young@unn.edu.ng \\ ${ }^{2}$ Department of Physiology University of Nigeria, Enugu Campus \\ chinyere.okafor@unn.edu.ng
}

\begin{abstract}
The development of new drugs and medical devices takes many years and a lot of scientific research. After identifying a new molecule, which is likely to be beneficial in human disease, it must undergo rigorous trials to ensure its efficacy and safety. Regulatory bodies ensure that clinical trials are carried out in a uniformly acceptable manner, and that the results are reliable. Their main aim is to protect the individual from harm. The aim of this review article is to describe the process of drug development and clinical trials as well as the role(s) of the regulatory agencies involved. A MEDLINE search was conducted using relevant keywords to access published information on clinical trials. Other sources of information include relevant websites and publications of regulatory agencies and major textbooks. The development of a new drug or device starts from identification of the molecule in the laboratory, where it is postulated to have useful characteristics. Following rigorous animal tests, the drug is then entered into clinical trials using human subjects. Clinical trials are conducted in four phases. Regulatory agencies are involved at every stage to ensure compliance with guidelines. There are various designs of clinical trials that may be used and the appropriate design has to be chosen to produce reliable results. The appropriate statistics must also be chosen and the submission to regulatory bodies for approval must be done in a systematic and scientific manner. The International Conference on Harmonisation was convened in 1990 and has drafted good clinical practice (GCP) guidelines for clinical trials. Submission of the application for a new drug involves proper regulatory writing procedures and protocols.
\end{abstract}

Keywords: clinical trial, regulatory agencies, ICH

\section{Introduction}

The development of new drugs and medical devices is an on-going process. It takes many years and a lot of scientific research to identify a new molecule, which is beneficial in human disease. The development of drugs and devices is a tightly regulated process. Clinical trial is an investigation in humans anticipated to decide or confirm the effects of a drug or to identify any adverse reactions with an intention of ascertaining its safety and efficacy [1]. Clinical trials are conducted under strict guidance from regulatory agencies. Regulatory agencies, though different in each continent, operate under similar principles with the ultimate aim of protecting the individual from harm. Good Clinical Practice (GCP) is the universal ethical and scientific quality standard for conducting clinical trials, and it applies to every aspect of the clinical trial process [2]. Thus GCP is applied from the stage of design, reporting and regulation of clinical trials.

There are scientific and regulatory rationales for conducting clinical trials. The first scientific rationale is that clinical trial medicine (CTM) helps to objectively decipher the usefulness or lack of it of a drug, device or procedure in a scientific and objective manner. The second rationale is that CTM eliminates the bias that occurs relying on informal observations or the results of non-randomized studies. It does this by avoiding the placebo effect and standardizing trial groups. Other scientific rationale for CTM is that it avoids the hazards of multiple post-hoc analyses of different trials, which will yield varying and 
South American Journal of Clinical Research

Volume 3, Issue 1, 2016

unreliable results. In addition, CTM helps to establish causation, avoiding spurious conclusions that are likely to be incorrect. It thus yields reliable information to guide clinicians. Clinical trials help to scientifically establish the efficacy and safety of clinical interventions such as drugs before they are registered for general public use.

Descriptive and Hypothesis testing are the two types of testing done in clinical trials [3]. There are various designs that may be used in the course of carrying out a clinical trial [4]. The choice of the appropriate design depends on several factors, including the phase of the trial, the drug, the target population etc. The appropriate choice of statistics is also crucial to the reliability or validity of the results. Clearly defined endpoints must be made, and the submission to regulatory bodies for approval must be done in a systematic and scientific manner. A surrogate endpoint may be chosen in place of a primary endpoint to enhance the efficiency of the study [5].

\section{Definition of clinical trial}

Clinical trial medicine is the science of designing, conducting and interpreting clinical trials [4]. Clinical trials are usually carried out in four phases [6];

Phase I: The drug is tested on a small number of healthy volunteers to determine appropriate dose, side effects etc. This phase tests the safety and maximum tolerated dose of a drug, its pharmacokinetics and pharmacodynamics in humans and drug-drug interactions. It is done in a small group of healthy and/or diseased volunteers.

Phase II: This is also referred to as 'therapeutic exploratory trial'. The drug is tested on a small number of actual patients. This phase tries to determine optimal doses, route and frequency of administration and endpoints. At the end of the Phase II trial, a preliminary meeting may be held between the sponsor, investigator and FDA to determine the feasibility and process of conducting a Phase III trial of the experimental drug [4].

Phase III: The drug is tested on a large numbers of patients. In this stage of drug assessment, the trial is conducted in a larger and often more diverse target population in order to demonstrate and/or confirm efficacy and to identify and estimate the incidence of common adverse reactions. Following a successful Phase III trial, a new drug application is made to the FDA. The Phase III trial may be a comparative efficacy (superiority or placebocontrolled) trial or an equivalency trial. The equivalency trial is usually preferred to avoid ethical issues that may arise from the use of placebo in a study [7]. A non-inferiority study may be conducted to demonstrate that the experimental drug is at least as effective as the usual or known standard treatment by a pre-specified margin. This type of trial however is more susceptible to false-positive results [8].

Phase IV: Post-marketing survey. This is required by the FDA to continue to monitor the drug for adverse effects. This is important because the Phase III trial may not have the power to detect adverse effects of less than 1 in 100 persons, as the trial is often conducted in between 300 to 3000 subjects [9]. However some literature have suggested that many phase IV studies are not actually conducted or completed by the companies [10].

\section{Hypothesis development and proposal writing}

A hypothesis is an untested scientific statement or observation [4]. Every study requires a well-defined hypothesis, which is then subjected to scientific testing. In most clinical trials, the hypothesis is that a new drug, device or procedure is able to perform a particular function or achieve a desired endpoint in an individual under certain defined circumstances, usually with minimal harm, or a clear evidence of the benefits outweighing the risks. A well-defined hypothesis is clearly spelt out in a trial protocol. The protocol is a document that describes how a clinical trial will be conducted. It details the objectives, design, methodology, statistical considerations and organization of a clinical trial. It also gives information on how the safety of the trial subjects and integrity of the data collected will be ensured [11]. 


\section{Design of clinical trials}

Certain parameters are critical when designing clinical trials;

a.) Design principles: a well-designed trial must abide by the following principles;

1. There should be internal and external validity.

2. Bias should be minimized.

3. Ensure that the right question is being asked; i.e. testing the right hypothesis.

b.) Design variables; these should be clearly worked out and defined. These include the appropriate target and sample population, the appropriate sample size, appropriate blinding procedure, determining the appropriate endpoint which must be significant, responsive to therapy, have low variability, and be representative of the disease status as a whole.

Trial designs: There are different types of designs used for clinical trials. Trials may be single arm design or multiple arm design [4]. A single arm design tests only one group of subjects. In this type of design, the differences between certain measures are checked before and after an intervention. Single arm designs are useful in homogenous populations, when the effect of the intervention is likely to be large and dramatic, and if appropriate patients are rare. It has limited ability to compare or demonstrate efficacy of interventions. A randomized controlled trial is regarded as the best type of trial, giving the most reliable result [5]. A randomized trial is able to eliminate selection bias that results from unknown or immeasurable confounders [6].

Multiple arm designs are more commonly used in clinical trials. They are usually parallel group designs in which patients are randomly assigned to two or more different and exclusive groups, each receiving a different treatment. One of the treatment arms may be assigned to placebo. In factorial designs, two or more different interventions are tested on the same group at the same time [13]. It can be two or more independent studies or a single study looking at interventions alone or in combination. Nested designs have one or more factors embedded within one or more other factors. Its also called cluster design or hierarchical design. It has multiple levels [14]. Sequential designs may be individual or group designs in which one group may be dropped or a drug dose modified. Other multi-period designs may be;

a.) Symptomatic vs disease-modifying interventions; It may be difficult in a trial to distinguish temporary from permanent effects of an intervention.

b.) Withdrawal designs; patients initially randomized to placebo or intervention during the first period, then all the patients are switched to placebo during the second period i.e. withdrawing the intervention.

In randomized withdrawal designs, all the patients are initially placed on an intervention. In the second period, the intervention is withdrawn from one arm and is replaced with placebo, while the other arm continues on the intervention. Carryover effects may affect withdrawal designs, if the initial treatment still lingers when the second phase is commenced. In practice, it may also be difficult or unethical to withdraw patients from treatment and place them on placebo.

Active extension design; in this trial design, patients are initially randomized to either an intervention or placebo. After a period, both groups are then continued on the intervention.

Randomized (staggered) start design; groups of patients are switched from placebo to intervention at different times. It can be done in a randomized fashion.

Using placebo in trials can be problematic and also lead to ethical issues. These can be minimized by;

1.) Continuing patients on effective treatment while introducing placebo as a second drug i.e. 'add-on design'. In add-on designs, patients are on effective treatment while the trial intervention is given as add-on or an extra measure.

2.) Minimizing the time patients are off known effective treatment; early escape design. Early escape designs require careful monitoring. Measures of disease progression must be convenient, reliable and accurate and the disease should be easy to monitor. 
Choosing the study sample: A sample is a representative of a larger population, which is selected for research [15]. There are different types of samples;

a) Haphazard sample; subjects are chosen in no particular way. The results are usually not useful.

b) Purposive sample; subjects that are selected are based on pre-defined criteria.

c) Convenience sample; involves selecting study subjects to suit the researcher.

d) Probability samples; usually more representative. Include simple random sample, systematic sample and cluster sample.

It is important to determine the type of sampling to be used, the study site and the study population as these are critical to the success of the trial. A poorly chosen sample will give results that are not representative of the larger population (poor external validity).

\section{Statistics in clinical trials}

The results of clinical trials, like scientific experiments, are subjected to statistical analysis. Statistical methods are used to summarize data, present the results in tabular or pictorial form and also to test the study hypothesis [15]. Statistics, when properly used can help to determine cause and effect relationships and throw more light on associations. However, if improperly used it may lead to the assumption of wrong inferences and conclusions. There are three main types of statistics. They include descriptive, inferential and comparative statistics [15].

1.) Descriptive statistics paints a picture of a situation, providing a concise numerical or graphical summary. It summarizes data sets and presents the results of a clinical trial in a simplified form. It could include diagrams and graphs.

2. Comparative statistics; It is a more sophisticated type of descriptive statistics that serves to compare in a numerical or graphical fashion one situation with another (or multiple other situations).

3. Inferential statistics; helps to suggest explanations for a situation or phenomenon. It allows you to draw conclusions based on extrapolations. Inferential statistics usually suggest but cannot absolutely prove an explanation or cause-and-effect relationship. Inferential statistics frequently involves estimation hypothesis testing.

The type of statistical method used depends on the variables measured during the trial. A variable is a measured characteristic or attribute that may assume different values. It may be quantitative or categorical. It may also said to be independent if its value is not determined by other variables and vice versa, in which case it is said to be a dependent variable. A statistical method that analyzes only one variable at a time is said to be univariate analysis, while analyzing multiple variables simultaneously is called multivariate analysis.

Inferential statistics; Hypothesis testing is useful in statistics to make conclusions. A hypothesis is a theory or suggestion for a phenomenon. Inferential statistics tests the null hypothesis. The null hypothesis (H0) is usually specified and either accepted or rejected using a specified significance level, which is either 0.05 or 0.01 . Two types of error may arise. A Type I error occurs if the null hypothesis is incorrectly rejected when it is actually true. Statistical tests may be one-tailed or two-tailed. A confidence interval is also useful. An x\% $\mathrm{CI}$ is a range, which should contain the value of a variable $\mathrm{x} \%$ of the time. It is common to use a $95 \%$ confidence interval.

The validity of any measurement is also important. Validity is the ability of a test to measure the phenomenon it is intended to measure [16]. A valid measure must be accurate and precise. Types of validity are face validity, construct validity, criterion validity and content validity. Construct validity is further sub-divided into convergent and discriminant validity. Convergent validity requires showing that parameters that are supposed to be related are found to be actually related, while discriminant validity means demonstrating that measures that are not supposed to be related are indeed unrelated. Criterion validity is also sub-divided into post-dictive validity, concurrent validity and predictive validity.

Measurements that are taken during a clinical trial also have to be accurate and reliable. Accuracy is how well a measure truly reflects the phenomenon being estimated, e.g. the 
accuracy of a weighing scale is how the weight recorded is truly representative of the patient's actual weight. Precision refers to how reproducible a measurement is with very little variation. It is also called reliability [16]. The simplest measure of precision is the standard deviation (SD). The higher the SD, the less precise the measurement is. The coefficient of variation is used to compare precision of different measurements even when they have different units. It is calculated as SD/mean. Statistically, the precision of a measured variable can be expressed as correlation coefficient, standard error of measurement, coefficient of variation, reliability limits of agreement, kappa coefficient etc.

\section{Documentation in clinical trials}

There are various forms of documents required during a clinical trial [17].

1. Investigator's brochure: Investigators' brochure is the reference book for the investigator. It has sections as specified by the International Conference on Harmonisation (ICH);

a.) Title page; this provides the sponsor's name, the identity of each investigational product and the release date.

b) Confidential statement; this states that the brochure should be treated as a confidential document only for the use of the investigator and the IRB/IEC.

c.) Table of contents

d.) Summary; this is usually brief and not exceeding two pages. It highlights the relevant physical, chemical, pharmacological, pharmaceutical, toxicological, pharmacokinetic, metabolic and clinical information available on the product.

e.) Introduction; this contains the chemical (generic, when approved) name of the investigational product, the rationale for the trial and the anticipated prophylactic, therapeutic or diagnostic indications.

Physical, chemical and pharmaceutical properties and formulation; this describes all the relevant properties of the compound being tested.

In non-clinical studies, the results of all relevant non-clinical pharmacology, pharmacokinetic, toxicology and metabolism of the investigational product should be outlined. The procedure carried out on the animals including the specie, number, sex etc. should be described. The effects of the product on humans i.e. pharmacokinetics, product metabolism, efficacy and safety should be documented.

Other types of documents include regulatory documents, which are submitted to relevant agencies to approve the drug.

\section{Protocol writing}

The purpose of a protocol is to define the objectives, population, study design, procedures, outcome variables and ethical conduct of product testing. The process of writing a protocol involves a good deal of team interaction and negotiation to reach a consensus. The International Conference on Harmonisation (ICH) E6 guideline describes the general content recommendations for protocol writing [18].

A protocol is a complete written description of and scientific rationale for a research activity involving human beings. There are three types of protocol;

1. Retrospective review.

2. Natural history study.

3. Interventional.

The protocol consists of the Title Page, Synopsis, Background information, Objectives and Methods; which consists of Study design, study population selection and withdrawal, outcome variables, study schedule, study monitoring, data management, statistical analysis and ethics. It may also be written with the aid of templates.

The clinical study report describes the results of a single human study. The ICH E3 guideline [19] describes the clinical study report. It should flow logically with an "IMRaD" structure. This consists of introduction, methods section, results description, and discussion. 
South American Journal of Clinical Research

Volume 3, Issue 1, 2016

\section{Regulatory documents}

The manufacturer of a product must provide written documentation called a 'Submission' about a product to a regulatory agency, making an argument for its safety and efficacy [17]. The required documents include source documents, integrated documents and global submissions. The use of templates and style guides are necessary in regulatory writing. Document templates are either instructional templates or property templates. Templates are useful and guide the format and content of a regulatory document. They may contain boilerplate text (which cannot be modified) or be totally blank. They facilitate the preparation of documents. They also ensure that there is consistent formatting, even though submissions are generated from different departments.

It is also important to use a style guide to answer questions that may arise. Style guides may be company-specific or standard. In general, the regulatory document should have a portrait page orientation; the text should be black with Times New Roman font and size 12. It should have 1.5-line spacing and the text should be justified for easy reading.

The code of federal regulations (CFR) is a list of regulations codified and divided into 50 titles, which are further divided into chapters, sub-chapters, parts and sections [20]. It is cited in the following order; Title, part and section. Some important sections of the CFR include;

INDs : 21 CFR 312, NDAs : 21 CFR 314, IRBs : 21 CFR 50 and 56.

Source documents are the first place where data are captured in a clinical trial. It consists of all written and printed documents that are pertinent to a research participant's exposure to the investigational agent(s), exposure to other treatments progress of the disease course and response to therapy.

The case report form (CRF) is the official clinical data-reporting document or tool used in a study [21]. It collects relevant data in a format compatible with the study protocol and regulatory requirements. The 3 major parts include the header, safety related modules and efficacy related modules. Each module is a block of specific questions and makes up a single page of the CRF. The Header must contain information on the Study No, Site No and Subject ID number. Safety modules are usually created from a standard library. They include demographics, adverse events, vital signs, medical history etc. Efficacy modules are designed for each therapeutic area based on the study protocol. Standard CRFs are necessary to allow consistent reporting across protocols and projects. CRF development is an interdisciplinary process and should include relevant members of the project team involved in the conduct, analysis and reporting of the trial. It begins as early as possible in the study preparation process. If a CRF is properly designed, its components will be reusable and save time and money. CRF is also used for subject tracking, data analysis and reporting, reports to FDA on safety etc.

An investigational new drug application (IND) may be for a treatment IND or an emergency IND [22]. It is the responsibility of a sponsor to submit an original and 2 copies of the IND application to the FDA. The IND is in effect 30 days after FDA receipt of application, unless FDA notifies the sponsor otherwise. If there are potential risks to subjects' safety and welfare, disapproval is rendered in writing by the FDA.

The contents of an IND application include; Cover sheet/application form (Form FDA1571), Table of contents, Introductory statement, General investigational plan, Investigator brochure, Protocol(s), Chemistry, manufacturing \& control info. Others include pharmacology and toxicology info, previous human experience with drug and other application information.

The application form is the FDA 1571. The form is provided for basic information like name of drug, submission date, sponsor identification, phase of proposed clinical investigation, sponsor commitments, identification of clinical monitor and safety evaluator, information regarding transfer of responsibilities to a contract research organization.

The FDA form 3500A is used to report adverse events of varying severity. Annual reports are also required on INDs and are due within 60 days of the anniversary of the IND. 
The Investigational Medicinal Products Dossier (IMPD) is a group of scientific documents required to make a clinical trial application to the European Union [23]. The general structure of the IMPD includes Quality (chemistry, manufacturing, and controls) data, Nonclinical pharmacology and toxicology data, Previous clinical trial and human experience data, and overall risk and benefit assessment.

The Integrated Summary of Safety (ISS) and Integrated Summary of Efficacy (ISE) are separate documents unique to regulatory submission for the United States. They are submitted to the Food and Drug Administration (FDA) in a New Drug Application (NDA) and are not required for European or Japanese submissions.

The writing up of regulatory documents is done as teamwork.

\section{Ethical issues}

Ethics is an important aspect of clinical trials. Prior to 1990, different countries had different regulations involving clinical trials and regulation of new products. The US Food and Drug Administration (FDA) agency, the European Union and Japan each had their own regulatory codes and framework for clinical trials [17]. In 1990, the first International Conference on Harmonization (ICH) was convened in Brussels and was composed of representatives from industry, academia and the governments of USA, EU and Japan [17]. Together, they drafted the first ICH good clinical practice (GCP) guidelines, which was adopted and has since undergone several revisions [24]. The last revision was done in 1997 AND produced Step 5. The ICH guidelines consist of eight sections viz;

1.) Glossary; this section defines the important terms in clinical research.

2.) The principles of ICH GCP; these consist of 13 basic tenets which are well-outlined in this section.

3.) Institutional Review Board(IRB)/Independent Ethics Committee(IEC): this section describes the composition, roles and responsibilities of the IRB/IEC.

4.) Investigators: describes the roles and responsibilities of the trial investigator.

5.) Sponsor: defines the role and responsibilities of the sponsor of the clinical trial.

6.) Clinical trial protocol and protocol requirements.

7.) Investigator's brochure.

8.) Essential documents for a clinical trial.

The IRB/IEC are groups that are available to protect the rights of patients participating in a clinical trial. The IRB is a group officially responsible for reviewing and monitoring biomedical research involving human subjects and imbued with the power to approve, require alterations in, or disapprove clinical studies [17]. The IRB reviews study protocols before the trial begins and monitors it as it progresses. It is a diverse group and consists of both scientists and non-scientists. It is also required to review and amend any protocol amendments.

There are other ethical regulatory agencies that ensure good ethical practices in the conduct of a clinical trial. The Data Safety Management Board (DSMB) is an independent committee specifically assembled to closely observe the study data throughout the duration of the study and look for any signs of scientific or ethical irregularities [25].

Another regulatory group is the Health Insurance Portability and Accountability Act (HIPAA). It helps ensure that electronic patient health, administrative and financial data is standardized, individuals, employers, health plans, and healthcare providers have unique identifying codes, and patient health information is secure and protected.

Drug approval process is a long and rigorous one. In the USA it is regulated by the FDA's Center for Drug Evaluation and Research (CDER).

\section{Regulatory agencies and processes}

Clinical trials are subjected to tight regulatory processes to safeguard the health and wellbeing of the members of the society. There are 13 basic tenets of a good clinical trial, which embodies the concept of GCP [17]. These are as follows; 
South American Journal of Clinical Research

Volume 3, Issue 1, 2016

1. Clinical trials should be conducted in accordance with the ethical principles that have their origin in the Declaration of Helsinki, and that are consistent with GCP and the applicable regulatory requirement(s).

2. Before a trial is initiated, foreseeable risks and inconveniences should be weighed against the anticipated benefit for the individual trial subject and society. A trial should be initiated and continued only if the anticipated benefits justify the risks.

3. The rights, safety, and wellbeing of the trial subjects are the most important considerations and should prevail over interests of science and society.

4. The available nonclinical and clinical information on an investigational product should be adequate to support the proposed clinical trial.

5. Clinical trials should be scientifically sound, and described in a clear, detailed protocol.

6. A trial should be conducted in compliance with the protocol that has received prior institutional review board (IRB)/independent ethics committee (IEC) approval/favourable opinion.

7. The medical care given to, and medical decisions made on behalf of, subjects should always be the responsibility of a qualified physician or, when appropriate, of a qualified dentist.

8. Each individual involved in conducting a trial should be qualified by education, training, and experience to perform his or her respective task(s).

9. Freely given informed consent should be obtained from every subject prior to clinical trial participation.

10. All clinical trial information should be recorded, handled, and stored in a way that allows its accurate reporting, interpretation, and verification.

11. The confidentiality of records that could identify subjects should be protected, respecting the privacy and confidentiality rules in accordance with the applicable regulatory requirement(s).

12. Investigational products should be manufactured, handled, and stored in accordance with applicable good manufacturing practice (GMP). They should be used in accordance with the approved protocol.

13. Systems with procedures that assure the quality of every aspect of the trial should be implemented.

The concept of good manufacturing process is also important in the development and production of drugs, devices and products for human consumption. Good manufacturing practice (GMP) is a set of regulations for manufacturers, processors and packagers of drugs, medical devices, some types of food and blood. These regulations help to ensure safety, purity and effectiveness of products. Some countries such as USA, Japan, Australia etc. have their own specific GMP requirements. Countries which do not have specific GMP requirements are required to use World Health Organisation (WHO) GMP guidelines. There are agencies which regulate GMP e.g FDA in USA, Therapeutical Goods Administration (TGA) in Australia, National Agency for Food, Drug and Control (NAFDAC) in Nigeria.

GMP requires clear documentation of processes and procedures by manufacturers. They must have quality assessment and control measures in place. There must be testing and validation of equipment and operations, in addition processes must be reliable with minimal variation. Pre-approval inspections are conducted before a drug or medical device is approved for marketing. Non-compliance with GMP regulations can lead to forfeiture of manufacturing rights, a fine or jail term.

In regulatory writing, the dossier is the collection or files of documents that contains all the technical data of pharmaceutical products to be approved, registered or marketed in the country. The common technical document (CTD) is a set of specification for applications dossier for the registration of medicines to be used in the USA, Europe and the rest of the world.

Regulatory affairs play a significant role at every stage of the drug development process; from the pre-clinical stage to the marketing of the product. Guidelines for clinical research 
have evolved over decades; from the Food, drug and cosmetics Act of 1938, the Nuremberg code of 1947, the Declaration of Helsinki in 1964 etc. There are protocols, reviews and decision-making at each step of the process of drug development. Every manufacturer must have a competent team that is aware of these regulations.

\section{The drug development process}

Drug development starts with in vitro tests in the laboratory, which is followed by animal studies (pre-clinical studies). Animal studies test the effects, distribution, metabolism and toxicity of drugs and involve at least two different species of animals. Both short-term and long-term testing is done. After the pre-clinical study, there will be a pharmacological profile of the drug.

An Investigational New Drug Application (IND) is then made to the CEDR. This application has to show that the drug is safe in animals and is also stable and consistent. The IND is evaluated within 30 days. This is then followed my clinical studies; including Phase I, Phase II and Phase III trials.

At the end of these, a New Drug Application (NDA) is made to the CEDR. At this stage, the CEDR determines if the application is 'fileable', and codes the type of drug entity; which may be a new molecular entity, a new salt or formulation of an existing drug, a combination of drugs, or an already marketed product. The CEDR then issues an action letter stating 'not approvable', 'approvable' or 'approved'.

There are two steps in the post-approval labelling, which include;

1.) Expanding indications and labelling. These occur following Phase IV trials, in which more information is provided about the drug in real life situations.

2.) Post-marketing surveillance involves monitoring after the drug has been approved and is in the market. This helps to identify situations where the drug is unsafe or not meeting demand.

\section{Conclusion}

Clinical trials are well-regulated scientific procedures used in the development of a new drug or device to be used in humans. The ICH ensures that there is protection of human subjects by providing a set of guidance rules for manufacturers and agencies. The whole process of drug development from the initial pre-clinical stage to the post-marketing stage is well documented and regulated. Regulatory bodies such as the FDA are made up of professionals who serve to ensure that each product released into the society is fit for human use.

\section{References}

[1.] Bellary S, Krishnankutty B, Latha MS. Basics of case report form designing in clinical research. Perspect. Clin Res 2014; 5(4): 159-166.

[2.] Bhatt A Quality of clinical trials: a moving target. Perspect. Clin Res 2011 ;2(4): 124-128.

[3.] Chernick M R, Friis R H. Introductory biostatistics for the health sciences. Modern applications including bootstrap. John Wiley \& Sons Inc. (2003).

[4.] Chin, R. Y., \& Lee, B. Y. (2008). Principles and practice of clinical trial medicine. Amsterdam: Elsevier/Academic Press.

[5.] Code of Federal Regulations Annual Edition. Available from https://www.gpo.gov/fdsys/browse/collectionCfr.action?collectionCode=CFR. Accessed $25^{\text {th }}$ March, 2016.

[6.] Data and Safety Monitoring Board Guidelines. Available from http://www.nidcr.nih.gov/Research/ToolsforResearchers/Toolkit/DSMBGuidelines.htm.

[7.] Eypasch E, Lefering R, Kum CK, Troidl H. Probability of adverse events that have not yet occurred: a statistical reminder. BMJ 1995; 311(7005): 619-620.

[8.] Fleming T R. Current issues in non-inferiority trials. Stat Med 2008; 27(3): 317-332. 
South American Journal of Clinical Research

Volume 3, Issue 1, 2016

[9.] Fontanarosa PB, Rennie D, DeAngelis CD. Post-marketing surveillance-lack of vigilance, lack of trust. JAMA. 2004; 292(21): 2647-2650.

[10.] Golafshani M. Understanding reliability and validity in qualitative research. The Qualitative Report 2003; 8(4): 597 - 606.

[11.] Guidance for industry E6 Good Clinical Practice: Consolidated Guidance. Available from http://www.fda.gov/downloads/Drugs/.../Guidances/ucm073122.pdf. Accessed 25th March, 2016.

[12.] https://hub.ucsf.edu/protocol-development.

[13.] Hulley SB, Cummings SR, Browner WS, Brady DG, Newman TB. Designing clinical research. Wolters Kluwer.

[14.] ICH Topic E3. Structure and content of clinical study reports. Available from http://www.ema.europa.eu/docs/en_GB/document_library/Scientific_guideline/2009/09/WC50000283 2.pdf.

[15.] Investigational New Drug (IND) Application. Available from http://www.fda.gov/drugs/developmentapprovalprocess/howdrugsaredevelopedandapproved/approvala pplications/investigationalnewdrugindapplication/default.htm. Accessed on $25^{\text {th }}$ June, 2016.

[16.] Johnson JJ, Gupta NV. Recent advances in quality management of clinical trials Int J Pharm PharmSci 2013:5(3): 34-38.

[17.] Montgomery AA, Peters TJ, Little P. Design, analysis and presentation of factorial randomised controlled trials. BMC Med Res Method. 2003 DOI: 10.1186/1471-2288-3-26.

[18.] Prentice R L. Surrogate endpoints in clinical trials: definition and operational criteria. Stat Med 1989; 8(4): 431-440.

[19.] Sibbald B, Roland M Understanding controlled trials; Why are randomised controlled trials important? BMJ 1998; 316(7126): 201.

[20.] The ICH harmonised tripartite guidelines. Available from

http://www.ich.org/fileadmin/Public_Web_Site/ICH_Products/Guidelines/Efficacy/E6/E6_R1_Guideli ne.pdf.

[21.] The Investigational Medicinal Products Dossier (IMPD). Available from http://www.impd.eu.

[22.] Tobin John J. Walsh G. Medical product regulatory affairs. Pharmaceuticals, Diagnostics, Medical devices.Wiley-VchVerlag GmbH \& Co. KGaA (2008).

[23.] Umscheid CA, Margolis DJ, Grossman CE. Key concepts of clinical trials; a narrative review. Postgrad Med (2011) Vol 123, No 5, pp 194-204.

[24.] Walker E. Nowacki AS. Understanding equivalence and non-inferiority testing. J Gen Intern Med 2011; 26(2): 192-196.

[25.] Yang LJS, Chang KWC, Chung K C. Methodology rigors in clinical research. Plast Reconstr Surg 2010; 129(6): 979e-988e. 